\title{
Giant Brunner's Gland Hamartoma of the Duodenal Bulb Presenting with Upper Gastrointestinal Bleeding and Obstruction
}

\author{
Ju Hyoung Lee, Kyeong Min Jo, Tae Oh Kim, Jong Ha Park, Seung Hyun Park, Jae Won Jung, So Chong Hur and Sung Yeun Yang \\ Division of Gastroenterology, Department of Internal Medicine, Inje University Haeundae Paik Hospital, Inje University College of Medicine, \\ Busan, Korea
}

Brunner's gland hamartomas are small benign lesions that are most commonly found in the bulb of the duodenum. They are very uncommon, and most are found incidentally during upper gastrointestinal series or esophagogastroduodenoscopy. The lesions tend to be asymptomatic, but patients may present with symptoms of duodenal obstruction or hemorrhage secondary to ulceration. Histologically, a Brunner's gland hamartoma consists of the components of Brunner's gland cells, as well as glandular, adipose and muscle cells. In this study, we report the case of a 30-year-old man who presented with upper gastrointestinal bleeding and obstructive symptoms due to a giant Brunner's gland hamartoma in the duodenal bulb. The hamartoma was successfully removed by endoscopic resection. No significant complications were observed. Microscopically, the lesion was found to be entirely composed of variable Brunner's glands and adipocytes. Clin Endosc 2016;49:570-574

Key Words: Brunner glands; Hamartoma; Gastrointestinal bleeding; Gastrointestinal obstruction

\section{INTRODUCTION}

Brunner's gland hamartoma, also known as Brunner's gland adenoma or brunneroma, is a rare benign tumor arising from the Brunner's glands of the duodenum. ${ }^{1}$ Brunner's gland hamartomas account for $10.6 \%$ of all benign tumors of the duodenum, and have been found in $0.008 \%$ of a single series of 215,000 autopsies. ${ }^{1,2}$ These lesions typically form polypoid pedunculated masses as they grow. The majority of Brunner's gland harmatomas are asymptomatic; however, in symptomatic patients, they can be treated by endoscopic or surgical excision. We report a case of the successful endoscopic removal of a large Brunner's gland hamartoma in a patient who pre-

Received: December 21, 2015 Revised: August 11, 2016

Accepted: August 25, 2016

Correspondence: Sung Yeun Yang

Division of Gastroenterology, Department of Internal Medicine, Inje University Haeundae Paik Hospital, Inje University College of Medicine, 875 Haeun-daero, Haeundae-gu, Busan 48108, Korea

Tel: +82-51-797-0200, Fax: +82-51-797-0298, E-mail: ysydrim@gmail.com

(cc) This is an Open Access article distributed under the terms of the Creative Commons Attribution Non-Commercial License (http://creativecommons.org/ licenses/by-nc/3.0) which permits unrestricted non-commercial use, distribution, and reproduction in any medium, provided the original work is properly cited. sented with upper gastrointestinal bleeding and obstruction.

\section{CASE REPORT}

A 30-year-old man presented with a 3-month history of nausea, bloating, dizziness, dyspnea on exertion, and self-limiting episodes of melena. His past medical history included obesity, hypertension, diabetes mellitus, hypercholesterolemia, and metabolic syndrome. He was initially seen at a primary clinic. A subepithelial duodenal lesion was detected on esophagogastroduodenoscopy, and this was diagnosed as a lipoma on endoscopic ultrasonography. He was managed conservatively, and received two 8-unit blood transfusions for his iron deficiency anemia. Subsequent abdominal ultrasound and colonoscopy findings were normal. However, the patient did not improve over the next 3 months and complained of persistent dizziness and nausea.

He was then referred to our hospital for further investigation. On questioning, he denied weight loss or the use of nonsteroidal anti-inflammatory drugs. His height and weight were $180.9 \mathrm{~cm}$ and $145.8 \mathrm{~kg}$, and he had a body mass index of 

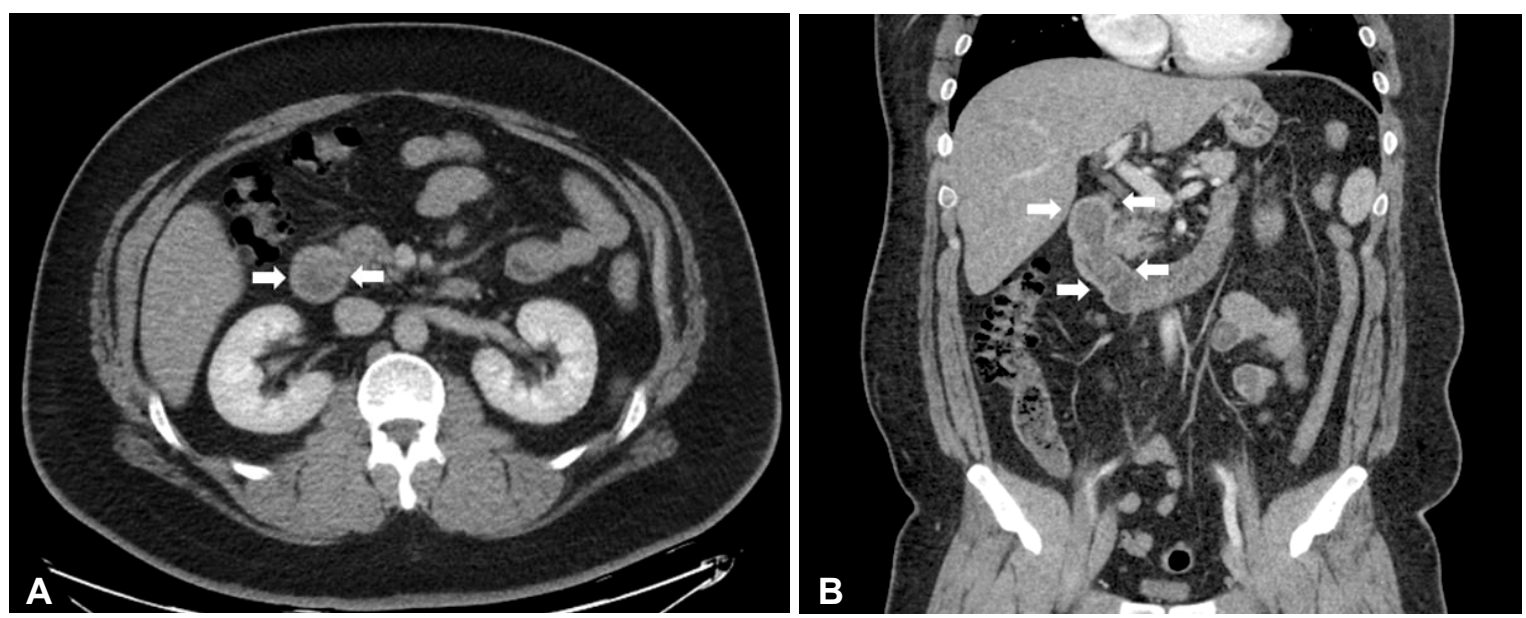

Fig. 1. (A) Contrast-enhanced axial and (B) coronal computed tomography scan shows a large polypoid mass in the duodenal bulb extending to second part of the duodenum (arrows).
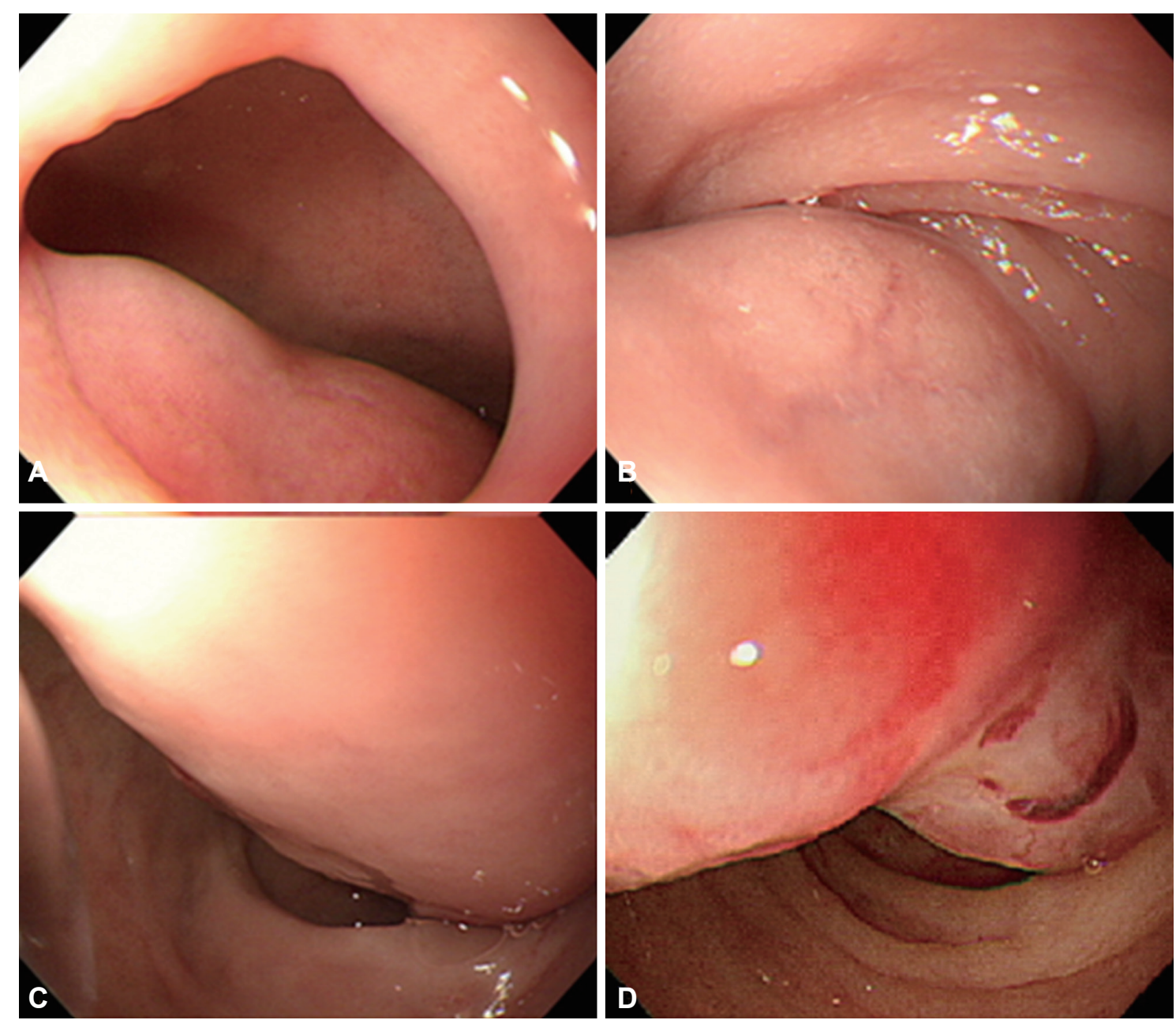

Fig. 2. Esophagogastroduodenoscopy shows a tortuous pedunculated mass occupying the lumen of the duodenal bulb and the second part of the duodenum. (A) Base of the tumor. (B) Thick trunk of the tumor at duodenal bulb. (C) Thick trunk at superior duodenal angle. (D) Tip of the tumor at the second part of the duodenum.

$44.5 \mathrm{~kg} / \mathrm{m}^{2}$. Physical examination did not reveal any abdominal tenderness, rebound tenderness, or palpable masses. His complete blood count was white blood cells 9,770/ $\mu \mathrm{L}$, hemo- globin $9.7 \mathrm{~g} / \mathrm{dL}$, hematocrit $31.6 \%$ (mean corpuscular volume $71.8 \mathrm{fL}$, mean corpuscular hemoglobin $22 \mathrm{pg}$ ), and platelets $281,000 / \mu \mathrm{L}$. Serum total bilirubin was $1.2 \mathrm{mg} / \mathrm{dL}$, serum as- 

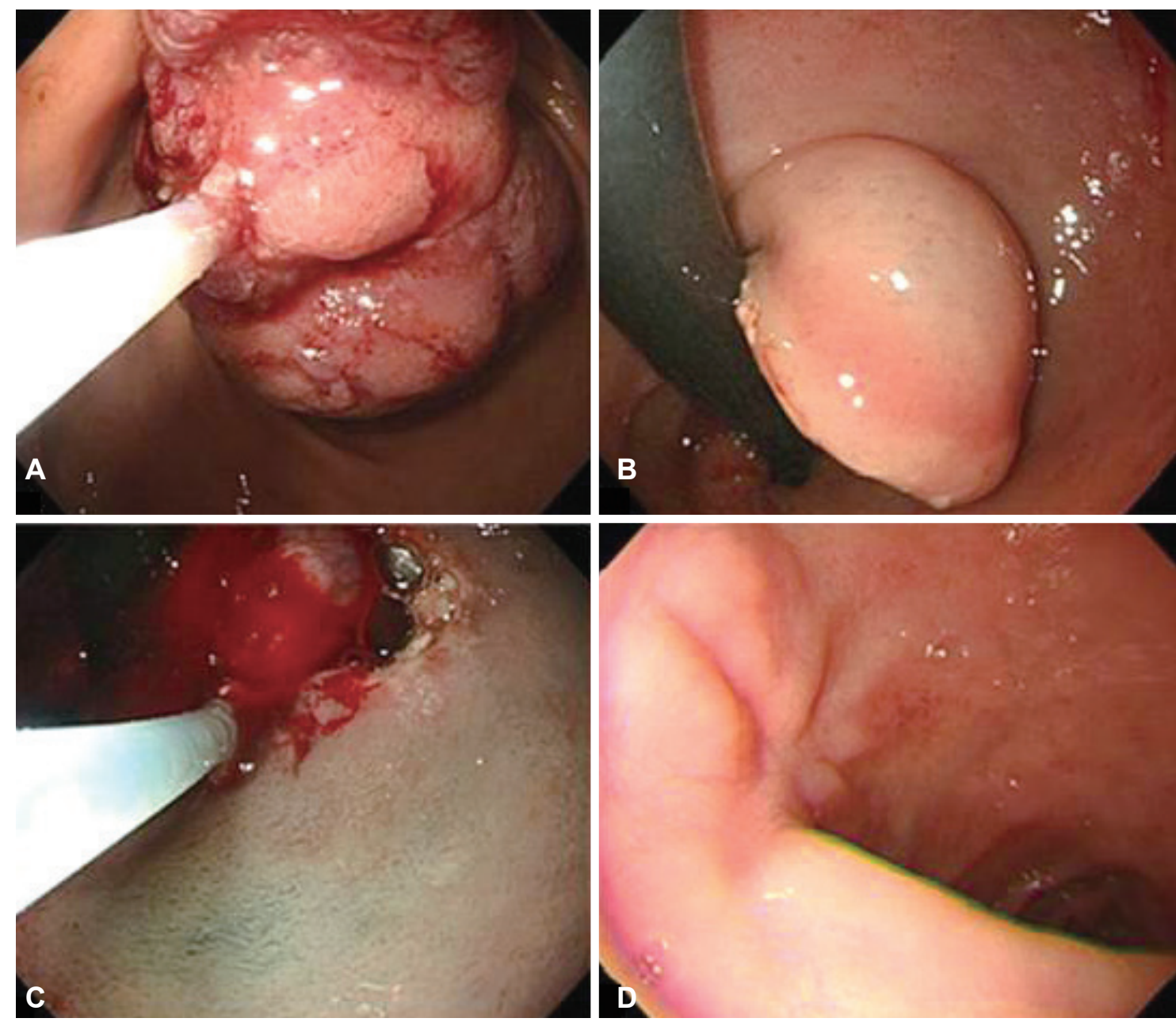

Fig. 3. Endoscopic removal. This shows the polyp being cut by two partial snare polypectomies and endoscopic submucosal dissection. It is successfully resected without any complications such as bleeding or perforation. (A) Stomach side. (B) Duodenal U-turn view. (C) Endoscopic submucosal dissection after U-turn. (D) Follow-up 2 months later.

partate aminotransferase was $37 \mathrm{IU} / \mathrm{L}$, and serum alanine transaminase was $67 \mathrm{IU} / \mathrm{L}$. Abdominal computed tomography showed a large polypoid mass in the duodenum (Fig. 1). It was a hypodense tubular intraluminal mass measuring about $9.3 \times 2$ $\mathrm{cm}$ in the first and second parts of the duodenum. Esophagogastroduodenoscopy showed a large pedunculated and eroded mass with a thick stalk arising from the duodenal bulb. The tumor was located on the anterior surface of the duodenal bulb and had a wide base, causing near-total obstruction (Fig. $2 \mathrm{~A}-\mathrm{C})$. The tip of the tumor also was covered with exposed vessels and hematin, but was not actively bleeding (Fig. 2D). Endoscopic polypectomy was performed. However, the lesion was too large to fit into the conventional snare. We grasped a portion of the mass and pulled it up into the stomach with the snare, and then performed two partial snare polypectomies (Fig. 3A). Next, we performed endoscopic submucosal dissection on the bulb of the duodenum after the duodenal U-turn (Fig. 3B, C). Hemoclips were applied to prevent bleeding and perforation. The gross endoscopic resection specimen showed a large duodenal lesion measuring $9.3 \times 2 \mathrm{~cm}$ (Fig. 4A). Histology confirmed a Brunner's gland hamartoma (Fig. 4B, C).

The patient did not experience any serious complications from this procedure. He was started on proton pump inhibitor therapy and discharged after 7 days. His dizziness and melena resolved after the endoscopic procedure, and his anemia resolved after 1 month. At 4 months after discharge, his complete blood count had normalized. At 6 months, a repeat esophagogastroduodenoscopy did not find any evidence of recurrence or tumor remnants (Fig. 3D).

\section{DISCUSSION}

Brunner's gland hamartoma, also known as Brunner's gland adenoma or brunneroma, is a rare benign tumor arising from the Brunner's glands of the duodenum. ${ }^{1}$ Brunner's glands were 

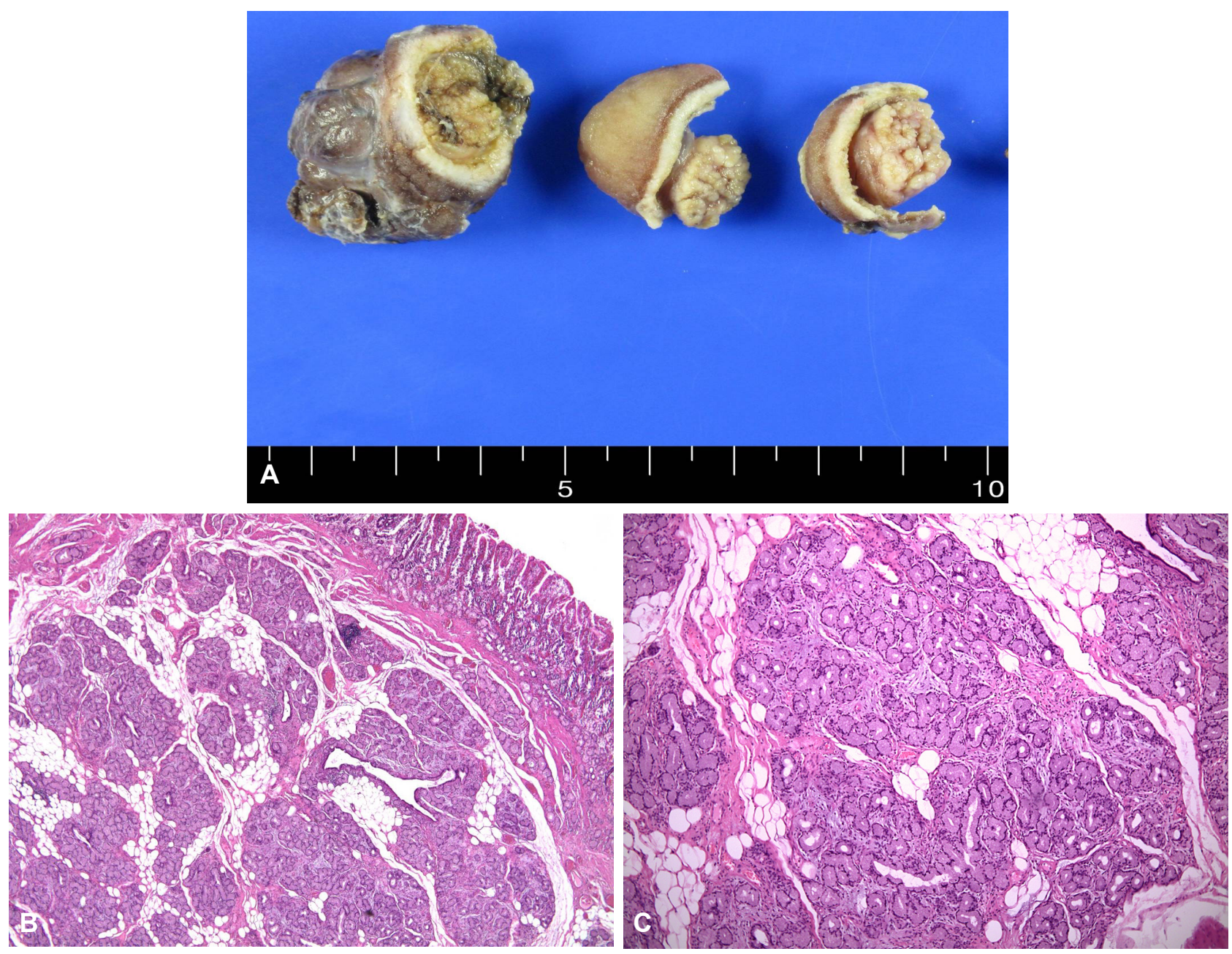

Fig. 4. Gross findings after formalin fixation. (A). A well-defined heterogenous yellow-white solid duodenal mass can be observed on the specimen section. The gross endoscopic resection specimen showed a large duodenal lesion measuring $9.3 \times 2 \mathrm{~cm}$. (B) Microscopic findings. Light microscopy revealed hyperplastic lobules of proliferating Brunner's glands separated by fibrous septum. (H\&E stain, $\times 40)$. (C) Brunner's gland hyperplasia composed of variable size of Brunner's glands (H\&E stain, $\times 100)$ can be observed.

first described by Brunner in 1688. These glands are branching actinotubular glands that arise in the duodenal submucosa. They secrete urogastrone, pepsinogen, and mucus to inhibit acid secretion and protect the duodenum from stomach acid. As such, they are crucial for preventing duodenal ulcers. The majority of these glands are seen in the first part of the duodenum, and they become smaller and less common towards the distal part of the duodenum. Their proportion in the duodenum decreases from $55 \%$ at birth to $35 \%$ by 50 years of age. ${ }^{2}$

Brunner's gland harmatomas typically develop into polypoid pedunculated masses as they enlarge. The exact pathogenesis is unknown. One hypothesis suggests that they may be related to irritation of the local lining, chronic pancreatitis, Helicobacter pylori infection, or parasympathetic activity. It is often difficult to differentiate between Brunner's gland hyperplasia and a Brunner's gland hamartoma. ${ }^{3}$ However, lesions smaller than $5 \mathrm{~mm}$, whether single or multiple, are defined as Brunner's gland hyperplasia, whereas lesions larger than 5 mm are termed hamartomas. ${ }^{4}$

Brunner's gland harmartomas are most commonly observed between the ages of 50 and 60 years, and the incidence is similar in both genders. ${ }^{5}$ Most patients are asymptomatic, but some may present with non-specific symptoms such as abdominal bloating, abdominal pain, and nausea. Brunner's gland harmartomas can be found incidentally during esophagogastroduodenoscopy or on imaging. ${ }^{6}$ Although symptomatic lesions are uncommon, patients can present with hemorrhage or obstruction. Gastrointestinal bleeding can occur because of ulcers or tumor erosion, causing in melena, iron deficiency anemia, fatigue, and in rare cases, hematemesis. ${ }^{5}$ They are mostly found in the duodenal bulb (57\%), which may be due to the vascular damage and stress from gastrointestinal motility present here. However, they can also be seen in the second (27\%) and third (7\%) parts of the duodenum. ${ }^{6}$ In this case, the 
patient was referred to our hospital after two episodes of large amounts of melena requiring blood transfusions. He was then found to have a large, elongated and pedunculated mass on the surface of the duodenal bulb during esophagogastroduodenoscopy.

Patients may experience obstruction when a mass becomes too large, or when there is diffuse hyperplasia. These patients often present with persistent nausea, vomiting, weight loss, epigastric pain, abdominal pain, and early satiety. Other uncommon symptoms include obstructive jaundice and intussusception due to the location and size of the tumor. ${ }^{4-6}$ These patients tend to have hamartomas exceeding $2 \mathrm{~cm}$ in size. In our patient, the Brunner's gland harmartoma had a thick stalk, which caused near-complete obstruction, resulting in the obstructive symptoms of nausea and vomiting. Several diagnostic tools including esophagogastroduodenoscopy and endoscopic ultrasound were used to identify the submucosal polypoid mass.

Brunner's gland harmartomas have characteristic histological features. $^{4-8}$ They consist of an unusual mix of normal tissue and Brunner's glands, adipose cells, lymphoid cells, as well as ductal tissue. Dysplasia is not observed.

The differential diagnosis for duodenal mass lesions include leiomyomas, adenomas, lipomas, adenocarcinomas, carcinoid tumors, lymphomas, leiomyosarcomas, pancreatic or ampullary tumors, and the lesions of Peutz-Jeghers syndrome.

Treatment depends on tumor size, symptoms, and the possibility of malignancy. Asymptomatic Brunner's gland hamartomas do not usually require treatment because their neoplastic potential is low. ${ }^{2}$ However, large or symptomatic lesions that cause obstruction or hemorrhage need to be removed. This can be done either endoscopically or surgically. Endoscopic treatment is preferred for pedunculated lesions; however, surgical resection is necessary if endoscopic methods fail, partic- ularly if there is a suspicion of malignancy.

In this present case, endoscopic removal proceeded successfully despite the size of the tumor. The mass was too large to fit into the conventional snare, and had to be pulled into the stomach and removed with two partial snare polypectomies. Following this, endoscopic submucosal dissection was performed after the U-turn in the duodenal bulb. This case shows that a non-malignant lesion can have significant clinical ramifications, and that Brunner's gland harmartomas can be treated successfully by careful use of endoscopic procedures.

Conflicts of Interest

The authors have no financial conflicts of interest.

\section{REFERENCES}

1. Nakabori T, Shinzaki S, Yamada T, et al. Atypical duodenal ulcer and invagination caused by a large pedunculated duodenal Brunner's gland hamartoma. Gastrointest Endosc 2014;79:679-680.

2. Jung Y, Chung IK, Lee TH, et al. Successful endoscopic resection of large pedunculated brunner's gland hamartoma causing gastrointestinal bleeding arising from the pylorus. Case Rep Gastroenterol 2013;7:304307.

3. Sen R, Gupta V, Sharma N, Chawla N, Kumar S, Malik S. Brunner gland hamartoma masquerading as malignancy: a rare case report. Middle East J Dig Dis 2014;6:237-240.

4. Gaspar JP, Stelow EB, Wang AY. Approach to the endoscopic resection of duodenal lesions. World J Gastroenterol 2016;22:600-617.

5. Lu L, Li R, Zhang G, Zhao Z, Fu W, Li W. Brunner's gland adenoma of duodenum: report of two cases. Int J Clin Exp Pathol 2015;8:7565-7569.

6. Martinez MA, Zyromski NJ, Luz LP. An unusual case of large symptomatic Brunner's gland adenoma: endoscopic ultrasound imaging. Endosc Ultrasound 2015;4:266-267.

7. Cheung TT, Ip EW, Poon RT, Trendell-Smith N. Brunner's gland adenoma: unusual cause of duodenal haemorrhage and obstruction. Hong Kong Med J 2013;19:460.e1-460.e2.

8. Stoos-Veic T, Tadic M, Aralica G. EUS-FNA of Brunner's gland hamartoma: a case report. Cytopathology 2013;24:194-196. 